\title{
Avaliação de amidos modificados empregados na mineração para aplicação em fluidos de perfuração de poços de petróleo
}

\author{
Evaluation of modified starches used \\ in the mining for application in oil \\ well drilling fluids
}

Sarah Suelen Simões Silva ${ }^{1}$, Karine Castro Nóbrega ${ }^{2}$, Luciana Viana Amorim ${ }^{1}$, Hélio de Lucena Lira ${ }^{2}$

\footnotetext{
${ }^{1}$ Unidade Acadêmica de Engenharia de Petróleo/Universidade Federal de Campina Grande/Campina Grande, Paraíba, Brasil.

e-mail: sarah_petroleo@outlook.com, luciana@cct.ufcg.edu.br

${ }^{2}$ Programa de Pós-Graduação em Ciência e Engenharia de Materiais/Universidade Federal de Campina Grande, Campina Grande, Paraíba, Brasil.

e-mail: karine.nobrega@hotmail.com, helio.lira@ufcg.edu.br
}

\section{RESUMO}

Amidos e seus derivados são utilizados em praticamente todos os setores industriais. Na indústria petrolífera podem ser empregados como aditivos controladores de filtrado, os quais controlam eficazmente a perda de fluido para a formação rochosa adjacente. O presente trabalho teve como objetivo avaliar o uso de amidos, de mandioca e de milho, utilizados na mineração, como redutor de filtrado em fluidos de perfuração poliméricos para poços de petróleo e gás. O estudo foi realizado em três etapas: na primeira etapa, cinco diferentes tipos de amidos modificados foram caracterizados por meio dos ensaios de viscosidade e índice de solubilidade em água; na segunda, as amostras de amido foram avaliadas de acordo com a norma da Petrobras (PE-2POC00727-0) e, na terceira e última etapa, as amostras que, atenderam as especificações da norma, foram avaliadas nas concentrações de 0 a $9 \mathrm{~g} / 350 \mathrm{~mL}$ de água, em uma formulação típica de fluido polimérico visando avaliar as propriedades reológicas e de filtração. Os dados obtidos foram comparados com um fluido polimérico aditivado com amido comercial. Os resultados demonstraram que dentre as cinco amostras estudadas, apenas a denominada de CMAN-AV foi considerada aprovada na norma e, quando adicionada ao fluido polimérico conferiu propriedades reológicas e de filtração semelhantes ao fluido aditivado com o amido comercial. Dessa forma, conclui-se que a amostra CMAN-AV pode ser utilizada como redutor de filtrado em fluido de perfuração polimérico.

Palavras-chave: amidos, redutor de filtrado, fluido polimérico.

\begin{abstract}
Starches and their derivatives are used in virtually all industrial sectors. In the petroleum industry the can be used as filtrate additives which effectively control the loss of fluid to the adjacent rock formation. The present work had as objective to evaluate the use of starches, of manioc and maize, used in the mining, as a filtrate reducer in polymeric drilling fluids for oil and gas wells. The study was carried out in three stages: in the first step, five different types of modified starches were characterized by the viscosity and water solubility index tests; in the second, the starch samples were evaluated according of with the of the Petrobras standard (PE-2POC-00727-0) and, in the third and last step, the samples that, attended the specifications of the standard, were evaluated in the concentrations of 0 to $9 \mathrm{~g} / 350 \mathrm{~mL}$ of water, in a typical formulation of polymeric fluid aiming to evaluate the rheological and filtration properties. The data obtained were compared with a polymer fluid additived with commercial starch. The results showed that among the five samples studied, only the called of CMAN-AV was considered approved in the standard and, when added to the polymeric fluid gaved rheological and filtration properties similar to the fluid additived with the commercial starch. Thus, it is concluded that sample CMAN-AV can be used as a filtrate reducer in polymeric drilling fluid.
\end{abstract}


Keywords: starches, filtrate reducer, polymeric fluid.

\section{INTRODUÇÃO}

Durante a perfuração de um poço os fluidos de perfuração à base de água ou óleo são bombeados da superfície para o fundo do poço e depois trazidos de volta para a superfície para remover os fragmentos de rocha (cascalhos) cortados pela broca. Após esse processo de limpeza, manutenção e ajuste das propriedades físicoquímicas do fluido, o mesmo recircula novamente no poço a fim de cumprir um conjunto de tarefas funcionais [1]. Como as formações rochosas subterrâneas de um poço são porosas e permeáveis, quando a pressão de formação é inferior à pressão hidrostática, ocorre a invasão da parte líquida do fluido (filtrado) para a formação que está sendo perfurada [2].

De acordo com GUO e PENG [3] e ALFORD et al. [4], a perda elevada de filtrado pode levar ao aumento do custo do fluido, formação de reboco excessivamente espesso, instabilidade do poço e danos à formação permeável.

As propriedades de filtração são consideradas uma das características mais importantes do fluido de perfuração. Uma das funções mais críticas dos fluidos é tentar minimizar a quantidade de filtrado que invade a formação porosa e permeável [5]. Para conseguir isto, foram desenvolvidos e aplicados agentes de controle de filtração incluindo materiais naturais, naturais modificados e totalmente sintéticos [3]. Os controladores de filtrado formam um reboco de baixa permeabilidade que impede a invasão da fase líquida do fluido para as formações permeáveis [6, 7].

O amido é um aditivo natural utilizado para controlar a perda de filtrado em fluidos aquosos preparados com água doce ou água saturada com sal [8]. Os polímeros do amido são formados por unidades de glicose arranjadas linearmente (amilose, com ligações $\alpha 1$-4) e ramificadas (amilopectina, com ligações $\alpha 1-4$ e a 1-6), variando as proporções de acordo com a fonte botânica. Grande parte dos amidos, como: milho, trigo, batata e mandioca, contêm de 18 a $28 \%$ de amilose [9]. Os amidos apresentam estabilidade térmica a cerca de $121^{\circ} \mathrm{C}\left(250^{\circ} \mathrm{F}\right)$ e estão sujeitas ao ataque bacteriano, exceto quando protegidos pela alta salinidade ou bactericida [8].

O amido é raramente utilizado pela indústria na sua forma nativa. A maioria dos amidos nativos apresentam aplicação limitada, tendo em vista que os mesmos são instáveis em relação às alterações de temperatura, $\mathrm{pH}$ e forças de cisalhamento e, apresentam também uma forte tendência para a decomposição e retrogradação [10].

Vários métodos foram desenvolvidos para produzir amidos modificados com uma variedade de características físico-químicas e aplicações [10]. A modificação é o processo de alteração das propriedades típicas do amido nativo por métodos químicos, físicos ou enzimáticos realizada com o objetivo de obter propriedades funcionais específicas [10-12].

Hoje, numerosos derivados de amido modificados estão comercialmente disponíveis para aplicações nos campos petrolíferos, incluindo principalmente amidos pré-gelatinizados, amidos reticulados, éteres e copolímeros de enxerto de amido solúveis em água [13]. O amido modificado é frequentemente utilizado no estágio inicial da perfuração para profundidades equivalentes à temperatura de fundo de poço de até $150^{\circ} \mathrm{C}$ [14].

Para obter amidos pré-gelatinizados, a gelatinização de amidos nativos é usualmente realizada em meio ácido ou alcalino. São necessárias temperaturas elevadas para quebrar as ligações de hidrogênio entre os grânulos de amido. A temperatura à qual ocorre a gelatinização depende da fonte do amido. Em amidos eterificados parte de grupos hidroxilo são substituídos por funções éter. O grau de modificação química pode ser expresso como o grau de substituição (DS), isto é, o número médio de substituintes por unidade Dglicose. Em comparação com os amidos nativos e os amidos pré-gelatinizados, estes produtos possuem boa solubilidade e mais grupos funcionais. Como exemplo de amido eterificado tem-se o hidroxipropilamido (HPA) e o carboximetilamido (CMS), comercializados também na indústria da mineração [13].

De acordo com pesquisas realizadas na literatura não há relatos de estudos relacionados à caracterização prévia de amidos modificados utilizados no setor da mineração para comprovação da sua eficácia como redutor de filtrado em fluidos de perfuração poliméricos. Muitos estudos relatam apenas o uso de amidos adquiridos no comércio local em fluidos aquosos, no entanto sabe-se que o conhecimento das propriedades físico-químicas do amido além da sua modificação permite entender as limitações funcionais desse polímero e ampliar o seu uso na formulação de fluidos de perfuração, os quais requerem funções específicas conforme variam as condições de reservatório e condições padrão de superfície. 
DANTAS et al. [15] verificaram que aditivos como amido, carboximetilcelulose de alta viscosidade (CMC HV) e calcita influenciaram as propriedades de filtração dos fluidos de perfuração inibidos. A utilização de amido e calcita em altas concentrações ou amido, CMC HV e calcita em altas concentrações $(12 ; 1,5$ e, $25 \mathrm{~g} / 350 \mathrm{~mL}$ de água, respectivamente) em fluidos aquosos, proporcionaram valores satisfatórios de perda de fluido final, aproximadamente $6 \mathrm{~mL}$, o que representa uma diminuição de cerca de seis vezes em relação ao fluido sem aditivos.

O potencial de ação do amido de milho na redução da perda de fluido durante as operações de perfuração foi confirmado nos resultados experimentais obtidos por GHAZALI et al. [2], tendo em vista que este amido por apresentar alta biodegradabilidade é considerado um aditivo adequado para ser utilizado no preparo de fluidos para perfuração das fases iniciais de poços onde a temperatura é relativamente baixa.

TALUKDAR e GOGOI [16] aplicaram um amido pré-gelatinizado (PGS) comercial em fluido de perfuração inibido e constataram que a faixa de concentração de 2,5 a 3,5g/100mL de água foi a ideal para que o PGS pudesse ser usado como aditivo redutor de filtrado, sem influenciar nas propriedades reológicas dos fluidos de perfuração formulados.

O ineficiente controle da filtração além de aumentar consideravelmente os custos inerentes a perfuração, pode resultar em dano a formação. A fim de otimizar as propriedades físico-químicas dos fluidos de perfuração no controle da filtração são utilizados aditivos controladores de filtrado como o amido modificado. Dessa forma, o presente trabalho se propôs em avaliar o uso de amidos, de mandioca e de milho, utilizados na mineração, como redutor de filtrado em fluidos de perfuração poliméricos para poços de petróleo e gás.

\section{MATERIAIS E MÉTODOS}

Para uma melhor compreensão, o presente estudo foi dividido em três etapas, conforme apresentado nos itens $2.1,2.2$ e 2.3

\subsection{Primeira Etapa: Caracterização das Amostras de Amido}

A caracterização das amostras de amido teve como objetivo conhecer as propriedades físicas (viscosidade e índice de solubilidade em água (ISA)) desses polímeros, a qual pode auxiliar na determinação da sua concentração no fluido polimérico e, inferir implicações dessas características na sua utilização como redutor de filtrado.

\subsubsection{Materiais}

Foram caracterizadas quatro amostras de amido de mandioca (CMAN-MV, CMAN-BV, CMAN-AV e PREMAN) e uma amostra de amido de milho (PRE-MILHO), as quais encontram-se nomeadas na Tabela 1. As amostras foram fornecidas pela Empresa System Mud an Imdex Limited Company, localizada na Rua Álvaro Beraldi, 421, Bairro Carvalho, Itajaí, SC.

Tabela 1: Identificação das amostras de amido caracterizadas.

\begin{tabular}{cc}
\hline TIPO DE AMIDO & NOMENCLATURA UTILIZADA \\
\hline Carboximetilamido de Mandioca de Média Viscosidade (CMS) & CMAN-MV \\
\hline Carboximetilamido de Mandioca de Baixa Viscosidade (CMS BV) & CMAN-BV \\
\hline Carboximetilamido de Mandioca de Alta Viscosidade (CMS AV) & CMAN-AV \\
\hline Amido Pré-gelatinizado de Mandioca & PRE-MAN \\
\hline Amido Pré-gelatinizado de Milho & PRE-MILHO \\
\hline
\end{tabular}

\subsubsection{Metodologia}

Para a determinação da viscosidade das diferentes amostras de amido foi utilizado o viscosímetro Brookfield (DV - II + Pro). Este equipamento é equipado com cilindros de diâmetros diferentes (spindles), em que é utilizado o cilindro adequado conforme a viscosidade do fluido. Para todas as amostras de amido em estudo foi utilizado o spindle 61, com exceção da amostra CMAN-AV, a qual teve sua viscosidade determinada utilizando o spindle 62. Para essa análise foram preparadas soluções poliméricas com diferentes concentrações $(2,5 ; 3,5 ; 4,5 ; 5,5 \mathrm{e}, 6,5 \mathrm{~g} / 250 \mathrm{~mL}$ de água deionizada) de amido, as quais permaneceram 5 minutos sob agitação constante, a uma velocidade de 17.000rpm no agitador mecânico Hamilton Beach, modelo 936. 
Para a determinação do índice de solubilidade em água, os amidos foram submetidos a diferentes temperaturas $\left(50,60,70,80\right.$ e $\left.90^{\circ} \mathrm{C}\right)$, conforme o método descrito por LEACH et al. [17] e ANDERSON et al. [18] com modificações. A análise foi desenvolvida em tubo falcão de fundo cônico de 50mL. A determinação se deu em uma suspensão de $250 \mathrm{mg}$ de amido em $20 \mathrm{~mL}$ de água destilada. Os amidos foram depositados em banho-maria (Ika HB 10 Digital) nas temperaturas citadas por 30 minutos. Durante o tempo de 30 minutos de aquecimento, realizou-se a cada 10 minutos a homogeneização, em agitador de tubos tipo vortex (Phoenix AP 56). Ao final do tratamento térmico, os tubos foram levados à centrífuga $(6000 \mathrm{rpm} / 24 \mathrm{minutos})$ para a separação de fases. Após a centrifugação, o material sobrenadante foi coletado e seco em estufa (Fanem Controlador Modelo $515 \mathrm{~A}$ ) a $100^{\circ} \mathrm{C}$ por $24 \mathrm{~h}$, até sua completa secagem para a quantificação da fração solúvel. O ISA foi calculado utilizando a Equação (1).

$$
\text { Índice de Solubilidade em Água }(\%)=\left(\frac{\text { massa solúvel }(g)}{\text { massa inicial de amido }(g)}\right) \times 100
$$

\subsection{Segunda Etapa: Avaliação das Amostras de Amido na norma PE-2POC-00727-0}

A avaliação das amostras de amido na norma PE-2POC-00727-0 [19] teve como objetivo analisar o desempenho desses polímeros de acordo com os procedimentos adotados pela mesma. Essa norma especifica as características exigíveis para qualificação, aceitação e método de ensaio para avaliação do redutor de filtrado para fluido de perfuração de base água na exploração e produção de poços de petróleo, em conformidade com Procedimentos, Diretrizes e Boas Práticas da Petrobras [19].

\subsubsection{Materiais}

Para formulação dos fluidos aquosos foram utilizados os seguintes aditivos químicos: cloreto de sódio $(\mathrm{NaCl})$, cloreto de cálcio $\left(\mathrm{CaCl}_{2}\right)$, cloreto de magnésio $\left(\mathrm{MgCl}_{2}\right)$, hidróxido de sódio $(\mathrm{NaOH}), 5$ amostras de amido e calcário aragonita micronizado $\left(\mathrm{CaCO}_{3}\right)$.

\subsubsection{Metodologia}

Foram preparados 9 fluidos de perfuração aquosos para a avaliação de cada amostra de amido segundo a norma PE-2POC-00727-0 [19]. Na Tabela 2 estão apresentados os aditivos e suas respectivas concentrações utilizados na formulação dos fluidos.

Tabela 2: Aditivos e suas respectivas concentrações utilizados na formulação dos fluidos aquosos [19].

\begin{tabular}{c|c}
\hline ADITIVOS & CONCENTRAÇÕES (g/350mL de água) \\
\hline $\mathrm{NaCl}$ & 21,0 \\
\hline $\mathrm{CaCl}_{2}$ & 0,07 \\
\hline $\mathrm{MgCl}_{2}$ & 0,028 \\
\hline $\mathrm{NaOH}$ & 1,0 \\
\hline $\mathrm{Amido}$ & 5,0 \\
\hline $\mathrm{CaCO}_{3}$ & 40,0 \\
\hline
\end{tabular}

Para a preparação dos fluidos, os aditivos foram adicionados de acordo com as concentrações e ordem apresentadas na Tabela 2. Em velocidade baixa (13.000rpm), os sais foram adicionados à água e permaneceram sob agitação por 15 minutos em agitador mecânico Hamilton Beach. Em seguida, foi adicionado o amido ao copo do misturador, por 1 minuto, agitando por mais 4 minutos em velocidade baixa. Por fim, foi adicionado o calcário ao copo do misturador, contendo a água, os sais e o amido, prolongando a agitação por 1 minuto em velocidade baixa.

Após o preparo do fluido, o mesmo foi vertido imediatamente para a célula do filtro-prensa API e deixado em repouso por 15 minutos (tempo considerado entre o final da agitação e o início da filtração). Após esse tempo de repouso foi aplicado imediatamente uma pressão de $345 \pm 35 \mathrm{kPa}(50 \pm 5 \mathrm{psi})$. A pressão foi mantida durante 30 minutos e ao final desse tempo todo o filtrado contido na proveta de $25 \mathrm{~mL}$ foi recolhido para análise baseando-se nos valores matemáticos (filtrado médio, desvio padrão e incerteza) calculados a partir das Equações (2), (3) e (4) respectivamente e, em requisitos (Tabela 3) exigidos pela norma PE-2POC00727-0 [19]. 
A média aritmética simples dos volumes de filtrado para os 9 ensaios executados foi calculada de acordo da Equação (2):

$$
V_{f}=\frac{1}{9} \sum_{i=1}^{9} V_{f i}
$$

Onde: $\mathrm{i}$ = subscrito que identifica cada ensaio.

O desvio padrão foi calculado de acordo com a Equação (3):

$$
\delta_{v}=\sqrt{\sum_{i=1}^{9} \frac{\left(V_{f i}-V_{f}\right)^{2}}{(n-1)}}
$$

Onde: $\delta_{\mathrm{v}}=$ desvio padrão dos volumes de filtrado; $\mathrm{V}_{\mathrm{fi}}=$ volume de filtrado de cada ensaio; $\mathrm{V}_{\mathrm{f}}=$ volume de filtrado médio; $\mathrm{n}=$ número de ensaios $=9$.

A incerteza foi calculada de acordo com a Equação (4):

$U\left(V_{f}\right)=\frac{2,366 \times \delta_{v}}{3}$

Onde: $\mathrm{U}\left(\mathrm{V}_{\mathrm{f}}\right)=$ incerteza da medida do volume de filtrado.

Tabela 3: Requisitos da norma PE-2POC-00727-0 [19].

\begin{tabular}{l|c|c}
\hline \multirow{4}{*}{ Filtrado $(\mathrm{mL})$} & MÉTODO ESTATÍSTICO & REQUISITOS (MÁXIMO) \\
\cline { 2 - 3 } & Média aritmética simples & 10 \\
\cline { 2 - 3 } & Incerteza & 2 \\
\hline
\end{tabular}

\subsection{Terceira Etapa: Avaliação das Amostras de Amido como Redutor de Filtrado em Fluidos Poliméri- $\cos$}

A avaliação das amostras de amido na formulação de fluidos de perfuração poliméricos teve como objetivo testar o desempenho da amostra CMAN-AV, a qual apresentou filtrado de acordo com os requisitos citados na norma PE-2POC-00727-0 [19] e, também da amostra de amido comercial (padrão) utilizada na indústria de petróleo.

\subsubsection{Materiais}

Para formulação dos fluidos poliméricos foram utilizados os seguintes aditivos químicos: anti-espumante, viscosificante (goma xantana $(\mathrm{GX})$ ), controlador de $\mathrm{pH}$ (óxido de magnésio $(\mathrm{MgO})$ ), redutores de filtrado (carboximetilcelulose de baixa viscosidade (CMC BV), carboximetilamido (CMAN-AV) e hidroxipropilamido de mandioca (HPA-Padrão)), sal $(\mathrm{NaCl})$, selante (calcita $\left(\mathrm{CaCO}_{3}\right)$ ) e bactericida (persulfato de sódio). Todos os aditivos, incluindo a amostra padrão de hidroxipropilamido de mandioca, foram fornecidos pela Empresa System Mud an Imdex Limited Company, localizada na Rua Álvaro Beraldi, 421, Bairro Carvalho, Itajaí, SC.

\subsubsection{Metodologia}

Os fluidos foram formulados com sete composições distintas, tendo a concentração de amido variando de 0 a 9g/350mL de água. De acordo com a Tabela 4, o fluido F1 foi formulado sem a presença de amido, os fluidos F2, F3 e F4 foram formulados com a amostra CMAN-AV e, os fluidos F5, F6 e F7 foram formulados com a amostra padrão.

Tabela 4: Formulações dos fluidos poliméricos.

\begin{tabular}{c|c|c|c|c|c|c|c}
\hline ADITIVOS (g/350mL de água) & F1 & F2 & F3 & F4 & F5 & F6 & F7 \\
\hline Anti-espumante & 0,4 & 0,4 & 0,4 & 0,4 & 0,4 & 0,4 & 0,4 \\
\hline $\mathrm{GX}$ & 1,5 & 1,5 & 1,5 & 1,5 & 1,5 & 1,5 & 1,5 \\
\hline $\mathrm{MgO}$ & 1,0 & 1,0 & 1,0 & 1,0 & 1,0 & 1,0 & 1,0 \\
\hline
\end{tabular}




\begin{tabular}{c|c|c|c|c|c|c|c}
\hline CMC BV & 3,5 & 3,5 & 3,5 & 3,5 & 3,5 & 3,5 & 3,5 \\
\hline Amido & - & 3,0 & 6,0 & 9,0 & 3,0 & 6,0 & 9,0 \\
\hline $\mathrm{NaCl}$ & 18,0 & 18,0 & 18,0 & 18,0 & 18,0 & 18,0 & 18,0 \\
\hline $\mathrm{CaCO}_{3}$ & 20,0 & 20,0 & 20,0 & 20,0 & 20,0 & 20,0 & 20,0 \\
\hline Bactericida & 0,01 & 0,01 & 0,01 & 0,01 & 0,01 & 0,01 & 0,01 \\
\hline
\end{tabular}

Os fluidos poliméricos foram preparados de acordo com a prática de campo, que consiste em adicionar os aditivos, um a um, sob agitação constante. Foi utilizado um agitador mecânico Hamilton Beach. Obedecendo a ordem descrita na Tabela 4, os aditivos foram adicionados a 350mL de água com intervalo de 10 minutos entre cada um deles, com exceção do anti-espumante que passou 5 minutos sob agitação constante a uma velocidade de 17.000rpm.

Para o estudo do comportamento reológico, após $24 \mathrm{~h}$ de repouso, o fluido foi agitado durante $5 \mathrm{~min}$ em agitador mecânico Hamilton Beach na velocidade de 17.000rpm. Em seguida, o fluido foi transferido para o recipiente do viscosímetro Fann modelo 35A. Inicialmente o viscosímetro foi acionado na velocidade de 600rpm durante 2 minutos e foi efetuada a leitura. Logo após, foi mudada para a velocidade de 300rpm, efetuando a leitura após 15 segundos.

Para obtenção da força gel inicial, o fluido foi submetido à velocidade de 600rpm durante 15 segundos, em seguida a velocidade foi alterada para 3rpm e o fluido ficou em repouso durante 10 segundos, então foi feita a leitura obtendo o valor da força gel inicial. Em seguida, para a obtenção da força gel final, o fluido foi deixado em repouso durante 10 minutos e efetuada a leitura na velocidade de $3 \mathrm{rpm}$.

Segundo a norma EP-1EP-00011-A [20], com os dados das leituras obtidas no viscosímetro, foi calculada a viscosidade aparente (VA) em cP, a viscosidade plástica (VP) em cP, o limite de escoamento (LE) em $\mathrm{N} / \mathrm{m}^{2}$ e, a força gel (FG) em N/m², utilizando as Equações (5), (6), (7) e (8), respectivamente.

$$
\begin{aligned}
& \mathrm{VA}=\frac{\mathrm{L}_{600}}{2} \\
& V P=L_{600}-L_{300} \\
& L E=L_{300}-V P \\
& F G=G_{f}-G_{o}
\end{aligned}
$$

Onde $\mathrm{L}_{600}$ é o valor obtido na leitura a 600rpm, $\mathrm{L}_{300}$ é o valor obtido na leitura a 300rpm, $\mathrm{G}_{\mathrm{f}}$ é o valor obtido do gel final e $\mathrm{G}_{\mathrm{o}}$ é o valor obtido do gel inicial.

$\mathrm{O}$ volume de filtrado (VF) foi determinado conforme a norma ANSI/API 13I [21] em filtro-prensa API. O fluido foi agitado por $1 \mathrm{~min}$ em agitação constante (17.000rpm), em seguida, o mesmo foi transferido para o recipiente do filtro-prensa e com aplicação de uma pressão na ordem de 690kPa (100psi) após 30 minutos o filtrado foi coletado em uma proveta graduada para análise.

A espessura do reboco (h) foi determinada com auxílio do extensômetro conforme a metodologia desenvolvida por FARIAS [22].

A permeabilidade do reboco $(\mathrm{k})$ foi determinada segundo a norma ANSI/API 13I [21], de acordo com a Equação (9).

$$
k=Q_{f} \cdot \varepsilon \cdot \mu \cdot 8,95 \times 10^{-5}
$$

Onde, $\mathrm{k}$ é dada em $\mathrm{mD} ; \mathrm{Q}_{\mathrm{f}}$ é o volume de filtrado, dado $\mathrm{em}^{3} \mathrm{~cm}^{3}$; $\varepsilon$ é a espessura do reboco, dada em $\mathrm{mm}$; e $\mu$ é a viscosidade da fase líquida do fluido, dada em $\mathrm{cP}$.

\section{RESULTADOS E DISCUSSÃO}

\subsection{Caracterização das Amostras de Amido}


A partir dos dados apresentados na Tabela 5, observa-se que o aumento da concentração de amido conferiu maior viscosidade as soluções e que a amostra CMAN-AV $(\mu=7210,0 \mathrm{cP})$, de acordo com as medidas de viscosidade provavelmente apresenta maior massa molecular em relação às demais amostras de amido estudadas. Segundo SPYCHAJ et al. [23], as diferentes massas molares e graus de substituição são fatores que influenciam a viscosidade das soluções aquosas de polímero.

O amido é constituído por cadeias de amilose e amilopectina. Em contato com água, a amilose é responsável pela função de gelificação, enquanto a amilopectina reduz os movimentos do polímero e aumenta a quantidade de conjuntos hidroxila dentro das moléculas de amido, expressando a propriedade hidrofílica dos amidos, possibilitando a sua dissolução em água. À medida que os grânulos continuam se expandindo, ocorre a lixiviação da amilose da fase intergranular para a fase aquosa, resultando no aumento substancial das propriedades reológicas da solução [24, 25]. Sendo assim, pode-se inferir que as amostras de amido estudadas apresentam teor de amilose crescente na seguinte ordem: CMAN-MV < PRE-MILHO < PRE-MAN < CMAN-BV $<$ CMAN-AV

Tabela 5: Medidas de viscosidade das amostras de amido.

\begin{tabular}{c|c|c|c|c|c}
\hline $\begin{array}{c}\text { CONCENTRAÇÃO DE AMIDO } \\
(\mathbf{g} / \mathbf{2 5 0} \mathbf{m L} \text { de água) }\end{array}$ & $\begin{array}{c}\boldsymbol{\mu}_{\text {CMAN-MV }} \\
(\mathbf{c P})\end{array}$ & $\begin{array}{c}\boldsymbol{\mu}_{\text {CMAN-BV }} \\
(\mathbf{c P})\end{array}$ & $\begin{array}{c}\boldsymbol{\mu}_{\text {CMAN-AV }} \\
(\mathbf{c P})\end{array}$ & $\begin{array}{c}\boldsymbol{\mu}_{\text {PRE-MAN }} \\
(\mathbf{c P})\end{array}$ & $\begin{array}{c}\boldsymbol{\mu}_{\text {PRE-MILHO }} \\
(\mathbf{c P})\end{array}$ \\
\hline 2,5 & 3,72 & 10,90 & 62,60 & 4,74 & 4,08 \\
\hline 3,5 & 3,84 & 13,10 & 152,70 & 5,34 & 3,96 \\
\hline 4,5 & 4,08 & 15,80 & 559,90 & 6,12 & 4,32 \\
\hline 5,5 & 4,02 & 18,80 & 2549,0 & 7,44 & 4,56 \\
\hline 6,5 & 4,14 & 22,70 & 7210,0 & 8,58 & 4,80 \\
\hline
\end{tabular}

Na Tabela 6 estão apresentados os valores do índice de solubilidade em água das amostras de amido estudadas. Amidos ricos em amilose mostram inchamento e solubilidade restritos, mesmo após período prolongado de aquecimento [26]. As diferenças do ISA na temperatura faixa de $50-90^{\circ} \mathrm{C}$ adotada para essa análise podem ser analisadas como resultado direto das diferenças do teor de amilose. As amostras CMANMV, CMAN-BV, PRE-MAN e PRE-MILHO, que provavelmente apresentam grânulos de amido com baixa quantidade de amilose são menos rígidos e absorvem mais água quando aquecidos em relação a amostra CMAN-AV.

De acordo com SINGH et al. [27], quando as moléculas de amido são aquecidas em excesso de água, a estrutura cristalina é rompida e, as moléculas de água formam pontes de hidrogênio entre a amilose e amilopectina, expondo seus grupos hidroxil, o que causa um aumento no inchamento e na solubilidade do grânulo. Esse poder de inchamento e solubilidade varia de acordo com a fonte do amido, fornecendo evidências da interação entre as cadeias de amido dentro dos domínios amorfos e cristalinos. A extensão destas interações é influenciada pela proporção amilose:amilopectina e pelas características dessas moléculas (peso molecular, comprimento de ramificações e conformação).

Tabela 6: Valores do índice de solubilidade em água das amostras de amido nas temperaturas de 50, 60, 70, 80 e $90^{\circ} \mathrm{C}$, expresso em $\%$.

\begin{tabular}{c|c|c|c|c|c}
\hline \multirow{2}{*}{ AMOSTRA } & \multicolumn{5}{|c}{ ÍNDICE DE SOLUBILIDADE EM ÁGUA (\%) } \\
\cline { 2 - 6 } & $\mathbf{5 0}^{\circ} \mathbf{C}$ & $\mathbf{6 0}^{\circ} \mathbf{C}$ & $\mathbf{7 0}^{\circ} \mathbf{C}$ & $\mathbf{8 0}^{\circ} \mathbf{C}$ & $\mathbf{9 0}^{\circ} \mathbf{C}$ \\
\hline CMAN-MV & 81,2 & 88,8 & 96,8 & 94,4 & 94,4 \\
\hline CMAN-BV & 91,2 & 88,8 & 96,0 & 98,4 & 98,8 \\
\hline CMAN-AV & 56,4 & 51,6 & 53,2 & 46,4 & 41,6 \\
\hline PRE-MAN & 80,0 & 90,0 & 91,2 & 93,6 & 94,8 \\
\hline PRE-MILHO & 95,2 & 100,0 & 98,8 & 100,0 & 98,8 \\
\hline
\end{tabular}


A solubilidade é consequência do lixiviamento da amilose. A redução na solubilidade observada apenas na amostra CMAN-AV indica que houve um fortalecimento das ligações, com o aumento nas interações entre 'as moléculas de amilose e amilopectina e entre as moléculas de amilopectina formando uma estrutura mais estável e reduzindo o lixiviamento das moléculas de amilose dos grânulos [28].

\subsection{Avaliação das Amostras de Amido na norma PE-2POC-00727-0}

Conforme análise dos resultados da Tabela 7 e baseando-se na norma PE-2POC-00727-0 [19], verificou-se que apenas uma das amostras de amido estudada, a CMAN-AV, foi considerada aprovada para uso como redutor de filtrado para poços de petróleo, visto que o filtrado médio máximo e a incerteza apresentaram valores de acordo com as especificações $\left(\mathrm{V}_{\mathrm{f}}<10 \mathrm{~mL}\right.$ e $\left.\mathrm{U}\left(\mathrm{V}_{\mathrm{f}}\right)<2\right)$. Esse resultado deve-se provavelmente a maior viscosidade apresentada pela amostra CMAN-AV.

Tabela 7: Variáveis analisadas para avaliar as amostras de amido.

\begin{tabular}{c|c|c|c|c|c}
\hline VARIÁVEIS & CMAN-MV & CMAN-BV & CMAN-AV & PRE-MAN & PRE-MILHO \\
\hline Filtrado Médio $-\mathrm{V}_{\mathrm{f}}(\mathrm{mL})$ & $>10$ & $>10$ & 5,62 & $>10$ & $>10$ \\
\hline Desvio Padrão $-\delta_{\mathrm{v}}$ & - & - & 0,3962 & - & - \\
\hline Incerteza $-\mathrm{U}\left(\mathrm{V}_{\mathrm{f}}\right)$ & - & - & 0,3125 & - & - \\
\hline
\end{tabular}

As amostras CMAN-MV, CMAN-BV, PRE-MAN e PRE-MILHO foram consideradas como produto reprovado, uma vez que o filtrado médio máximo foi superior ao valor de requisito.

\subsection{Avaliação das Amostras de Amido como Redutor de Filtrado em Fluidos Poliméricos}

A Tabela 8 apresenta as propriedades reológicas (Viscosidade Aparente - VA; Viscosidade Plástica - VP; Limite de Escoamento - LE e Força Gel - FG) obtidas para os fluidos de perfuração poliméricos estudados.

Tabela 8: Propriedades reológicas dos fluidos poliméricos estudados.

\begin{tabular}{c|c|c|c|c}
\hline FLUIDOS & VA $(\mathbf{c P})$ & VP $(\mathbf{c P})$ & $\mathbf{L E}\left(\mathbf{N} / \mathbf{m}^{2}\right)$ & FG $\left(\mathbf{N} / \mathbf{m}^{2}\right)$ \\
\hline F1 & 32,15 & 23,14 & 18,02 & 2,50 \\
\hline F2 & 61,15 & 34,64 & 53,02 & 5,16 \\
\hline F3 & 81,80 & 45,60 & 72,40 & 6,00 \\
\hline F4 F5 & 122,33 & 60,34 & 123,99 & 6,33 \\
\hline F6 & 43,25 & 33,00 & 19,50 & 1,75 \\
\hline F7 & 49,00 & 30,75 & 36,50 & 2,25 \\
\hline
\end{tabular}

O fluido F1 apresentou os menores valores de VA $(32,15 \mathrm{cP}), \mathrm{VP}(23,14 \mathrm{cP}), \mathrm{LE}\left(18,02 \mathrm{~N} / \mathrm{m}^{2}\right)$ e FG $\left(2,50 \mathrm{~N} / \mathrm{m}^{2}\right)$, comparado com os outros fluidos formulados com a amostra CMAN-AV, devido ao fato de não ter sido aditivado com amido.

Observa-se um aumento em todas as propriedades reológicas analisadas, quando da aditivação dos fluidos (F2, F3 e F4) com a amostra CMAN-AV, em comparação com o fluido F1. Essa amostra trata-se de um amido eterificado mais especificamente um carboximetilamido, sendo considerado um tipo de polieletrólito. Na solubilização de polieletrólitos em meio aquoso (hidratação) a dissociação dos grupos iônicos conferem a estes múltiplas cargas elétricas. Tais espécies químicas são denominadas de poliíons, que na repulsão eletrostática promovem o estiramento da cadeia polimérica, transformando a sua conformação de "enovelada" para "linearizada". E essa linearização da cadeia polimérica promove a viscosificação do meio aquoso [29].

O fluido F4 formulado com a concentração máxima da amostra CMAN-AV $(9,0 \mathrm{~g} / 350 \mathrm{~mL}$ de água) apresentou os valores mais elevados de VA $(122,33 \mathrm{cP})$, VP $(60,34 \mathrm{cP})$, LE $\left(123,99 \mathrm{~N} / \mathrm{m}^{2}\right)$ e FG $\left(6,33 \mathrm{~N} / \mathrm{m}^{2}\right)$ dentre todos os fluidos testados. Essas características reológicas estão relacionadas com as propriedades 
físico-químicas desse polímero. O carboximetilamido é um amido modificado quimicamente através do processo de eterificação, onde grupos hidroxila são substituídos por funções éter, conferindo aos amidos eterificados boa solubilidade e mais grupos funcionais, o que garante uma grande capacidade de absorção de água. A principal função desses polímeros é a de tornar o fluido mais viscoso, melhorando a capacidade de carregamento de cascalhos. Assim, como os polímeros naturais, os polímeros modificados são agentes hidrofílicos capazes de absorver grande quantidade de água [30].

Pode-se inferir também que o aumento da concentração da amostra CMAN-AV em 3,0; 6,0 e $9,0 \mathrm{~g} / 350 \mathrm{~mL}$ de água contribuiu para a aumento das propriedades reológicas. De acordo com ALASKARI e TEYMOORI [31], a adição de polímeros é responsável pela formação de cadeias complexas que aumentam a viscosidade do fluido e esse aumento é mais visível com o aumento da concentração do polímero.

A Tabela 9 apresenta os resultados referentes às propriedades de filtração (Volume de Filtrado (VF); Espessura do Reboco - h e Permeabilidade do Reboco - k) obtidas para os fluidos poliméricos estudados.

Tabela 9: Propriedades de filtração dos fluidos poliméricos estudados.

\begin{tabular}{c|c|c|c}
\hline FLUIDO & $\mathbf{V F}(\mathbf{m L})$ & $\mathbf{h}(\mathbf{m m})$ & $\mathbf{k}\left(\mathbf{1 0} \mathbf{-}^{-\mathbf{m}} \mathbf{)}\right)$ \\
\hline 1 & 9,4 & 0,779 & 0,655 \\
\hline 2 & 7,0 & 0,698 & 0,437 \\
\hline 3 & 6,1 & 0,938 & 0,512 \\
\hline 4 & 4,8 & 1,002 & 0,430 \\
\hline 5 & 9,2 & 1,220 & 1,000 \\
\hline 6 & 8,1 & 1,166 & 0,850 \\
\hline 7 & 6,0 & 0,820 & 0,440 \\
\hline
\end{tabular}

Observou-se que o fluido F1 apresentou maior valor de VF $(9,4 \mathrm{~mL})$ e $\mathrm{k}\left(0,655 \times 10^{-3} \mathrm{mD}\right)$ em comparação com os fluidos formulados com a amostra CMAN-AV, provavelmente devido a maior quantidade de água livre no sistema e ausência do aditivo polimérico em sua formulação.

Para efeito de comparação foram formulados fluidos de perfuração com a amostra padrão de hidroxipropilamido de mandioca (HPA-Padrão) nas concentrações citadas na Tabela 4. O Hidroxipropilamido é comercializado na indústria do petróleo como redutor de filtrado e, trata-se de um amido modificado quimicamente através da introdução de grupamentos de hidroxipropil a sua estrutura [32]. O fluido F4 formulado com concentração máxima $(9,0 \mathrm{~g} / 350 \mathrm{~mL}$ de água) da amostra CMAN-AV apresentou volume de filtrado com valor de $4,8 \mathrm{~mL}$, enquanto que na mesma concentração de HPA-Padrão no fluido F7, o valor de VF foi de 6,0mL. Essa diferença pode ser explicada devido diferentes faixas de viscosidade apresentadas por esses dois fluidos. Os fluidos F4 e F7 apresentaram valores de viscosidade de 122,33 e 59,75cP, respectivamente, o que mostra a grande disparidade nas características reológicas conferidas ao fluido pela adição dos diferentes polímeros. Segundo LEITE [33], o volume de filtrado também é influenciado pela viscosidade, maiores valores de viscosidades conduzem a menores valores de VF.

Com base na Tabela 9 foi observado também que os fluidos aditivados com a amostra CMAN-AV apresentaram valores crescentes de espessura de reboco (de 0,698 a 1,002mm) à medida que se aumentou a concentração do polímero no fluido, o que difere dos resultados apresentados na literatura. Segundo GHAZALI et al. [2] e BLKOOR e FATTAH [34], o aumento da espessura do reboco é inversamente proporcional ao aumento da concentração do polímero. Essa proporcionalidade apresentada entre concentração e espessura do reboco para os fluidos F2, F3 e F4, ocorreu provavelmente devido a viscosidade elevada da amostra CMAN-AV e a concentração mais elevada do polímero na formulação.

Os fluidos F2, F3 e F4 apresentaram valores próximos de permeabilidade, sendo estes valores de 0,437; 0,512 e 0,430x $10^{-3} \mathrm{mD}$, respectivamente. Quando comparados aos valores de permeabilidade apresentados pelos fluidos F5, F6 e F7, de 1,0; 0,85 e $0,44 \times 10^{-3} \mathrm{mD}$ respectivamente, observa-se que apesar dos fluidos aditivados com a amostra CMAN-AV apresentarem altas permeabilidades, estas são inferiores aos valores obtidos para os fluidos formulados com o amido padrão. A elevada permeabilidade neste caso está relacionada com os altos valores de h e VF, uma vez que a permeabilidade do reboco é determinada com bases 
nessas duas propriedades. Quanto maior a permeabilidade adquirida pelo reboco menor será o controle de filtração [33].

\section{CONCLUSÕES}

Com o objetivo de avaliar o uso de amidos, de mandioca e de milho, utilizados na mineração, como redutor de filtrado em fluidos de perfuração poliméricos para poços de petróleo e gás, pode-se concluir que:

- A amostra CMAN-AV apresentou maior viscosidade em relação as demais amostras de amido estudadas, resultado esse que pode estar relacionado a forte dependência da viscosidade em relação a massa molecular da cadeia polimérica;

- As amostras CMAN-MV, CMAN-BV, PRE-MAN e PRE-MILHO apresentaram aumento do ISA com o aumento da temperatura;

- Apenas a amostra CMAN-AV foi aprovada na norma PE-2POC-00727-0;

- O fluido F4 formulado com a concentração máxima da amostra CMAN-AV (9g/350mL de água) apresentou os valores reológicos mais elevados de VA $(122,33 \mathrm{cP})$, VP $(60,34 \mathrm{cP})$, LE $\left(123,99 \mathrm{~N} / \mathrm{m}^{2}\right)$ e FG $\left(6,33 \mathrm{~N} / \mathrm{m}^{2}\right)$ dentre todos os fluidos testados e, os menores valores VF $(4,8 \mathrm{~mL})$ e $\mathrm{k}\left(0,430 \times 10^{-3} \mathrm{mD}\right)$;

- Os fluidos formulados com a amostra CMAN-AV apresentaram propriedades reológicas e de filtração compatíveis as dos fluidos formulados com a amostra HPA-Padrão comercial.

Dessa forma, pode-se concluir ainda que a amostra CMAN-AV que se trata de um carboximetilamido de mandioca de alta viscosidade, apresenta potencial para ser utilizada como redutor de filtrado em fluidos de perfuração poliméricos para poços de petróleo e gás.

\section{AGRADECIMENTOS}

Os autores agradecem ao CNPq, Conselho Nacional de Desenvolvimento Científico e Tecnológico - Brasil, pelo apoio financeiro do programa PIBIC/CNPq-UFCG, ao Laboratório de Pesquisa em Fluidos de Perfuração (PeFLab) instalado no Laboratório de Referência em Dessanilização (LABDES) pelo uso de suas instalações físicas e apoio à pesquisa e, a Empresa System Mud na Imdex Limited Company/SC pelo fornecimento dos aditivos.

\section{BIBLIOGRAFIA}

[1] AMANULLAH, M., RAMASAMY, J., AL-ARFAJ, M. K., et al., “Application of an indigenous ecofriendly raw material as fluid loss additive”, Journal of Petroleum Science and Engineering, v. 139, pp. 191$197,2016$.

[2] GHAZALI, N. A., ALIAS, N. H., MOHD, T. A. T., et al., "Potential of corn starch as fluid loss control agent in drilling mud", Applied Mechanics and Materials, v. 754-755, pp. 682-687, 2015.

[3] GUO, W., PENG, B., "Synthesis and characterization of starch-graft-polyacrylamide copolymers and their application as filtration control agents in drilling fluid", Journal of Vinyl \& Additive Technology, v. 18, n. 4, pp. 261-266, 2012.

[4] ALFORD, P., ANDERSON, D., BISHOP, M., et al., "Novel oil based mud additive decreases HTHP fluid loss and enhances stability", In: AADE Fluids Technical Conference and Exhibition, Houston, Texas, 15-16 April 2014.

[5] NAGRE, R. D., ZHAO, L., OWUSU, P. A., "Thermosaline resistant acrylamide-based polyelectrolyte as filtration control additive in aqueous-based mud", Petroleum \& Coal, v.56, n. 3, pp. 222-230, 2014.

[6] SHERWOOD, J. D., MEETEN, G. H., "The filtration properties of compressible mud filtercakes", Journal of Petroleum Science and Engineering, v. 18, n. 1-2, pp. 73-81, 1997.

[7] LOMBA, R. Fundamentos de filtração e controle das propriedades de filtração: apostila. 26p, 2010.

[8] SCHLUMBERGER OILFIELD SERVICES, http://www.glossary.oilfield.slb.com/Terms/s/starch.aspx. Acessado em abril de 2018.

[9] ADAMOVICZ, J. A. L., ALBERTON, C., OLIVEIRA, C. S., et al., "Análise térmica, estrutural e reológica do amido de batata com adição de hidrocolóides”, In: IX Congresso Brasileiro de Análise Térmica e Calorimetria, Serra Negra, São Paulo, 09-12 Novembro 2014. 
[10] ALCÁZAR-ALAY, S. C., MEIRELES, M. A. A., "Physicochemical properties, modifications and applications of starches from different botanical sources", Food Science and Technology, v. 35, n. 2, pp. 215$236,2015$.

[11] RICHARDSON, S., GORTON, L., "Characterisation of the substituent distribution in starch and cellulose derivatives", Analytica Chimica Acta, v. 497, n. 1-2, pp. 27-65, 2003.

[12] LEWICKA, K., SIEMION, P., KURCOK, P., "Chemical modifications of starch: microwave effect", International Journal of Polymer Science, v. 2015, pp. 1-10, 2015.

[13] ZHANG, L., "A review of starches and their derivatives for oilfield applications in China", Starch/Stärke, v. 53, n. 9, pp. 401-407, 2001.

[14] AMANULLAH, M., YU, L., "Environment friendly fluid loss additives to protect the marine environment from the detrimental effect of mud additives", Journal of Petroleum Science and Engineering, v. 48, n. 3-4, pp. 199-208, 2005.

[15] DANTAS, A. P. T., LEITE, R. S., NASCIMENTO, R. C. A. M., et al., "The influence of chemical additives in filtration control of inhibited drilling fluids", Brazilian Journal of Petroleum and Gas, v. 8, n. 3, pp. 97-108, 2014.

[16] TALUKDAR, P., GOGOI, S. B., "A study on the role of pre-gelatinized starch (PGS) in the non damaging drilling fluid (NDDF) for the Tipam sand of Geleki oilfield of upper Assam Basin", International Journal of Applied Sciences and Biotechnology, v. 3, n. 2, pp. 291-300, 2015.

[17] LEACH, H. W., MCCOWEN, L. D., SCHOCH, T. J., "Structure of the starch granule. I. Swelling and solubility patterns of various starches", Cereal Chemistry, v. 36, n. 6, pp. 534-544, 1959.

[18] ANDERSON, R. A., CONWAY, V, F., GRIFFIN, E. L., "Gelatinization of corn grits by roll-and extrusion-cooking”, Cereal Science Today, v. 14, n. 1, pp. 4-7, 1969.

[19] PETROBRAS, Redutor de filtrado para poços de petróleo, PE-2POC-00727-0, 2017.

[20] PETROBRAS, Viscosificante para fluidos usados na exploração e produção de poços de petróleo e gás, EP-1EP-00011-A, 2011.

[21] ANSI/API, Recommended practice for laboratory testing of drilling fluids 13I, Eighth Edition, 2009.

[22] FARIAS, K. V., Influência de umectante aniônico na reologia e espessura do reboco de fluidos hidroargilosos, Dissertação de M.Sc., PRH ANP - 25/UFCG, Campina Grande, PB, Brasil, 2005.

[23] SPYCHAJ, T., WILPISZEWSKA K., ZDANOWICZ, M., "Medium and high substituted carboxymethyl starch: Synthesis, characterization and application”, Starch $\square$ Stärke, v. 65, n. 1-2, pp. 22-33, 2013.

[24] VANDEPUTTE, G. E., VERMEYLENA, R., GEEROMS, J., et al., "Rice starches. I. Structural aspects provide insight into crystallinity characteristics and gelatinisation behavior of granular starch", Journal of Cereal Science, v.38, n. 1, pp. 43-52, 2003.

[25] BERGTHALlER, W., HOLlMANN, J. “Comprehensive glycoscience”, In: Hans, K. (ed), Starch, p. 579-612, Oxford: Elsevier, 2007.

[26] OLIVEIRA, T. M., PIROZI, M. R., BORGES, J. T. S., et al., "Caracterização do amido de grão-de-bico (Cicer arietinum L.)", Boletim do Centro de Pesquisa de Processamento de Alimentos, v. 27, n. 1, pp. 27-42, 2009.

[27] SINGH, N., SINGH, J., KAUR, L., et al., "Morphological, thermal and rheological properties of starches from different botanical sources", Food Chemistry, v. 81, n. 2, pp. 219-231, 2003.

[28] ZAVAREZE, E. R., RODRIGUES, A. O., STORCK, C. R., et al., "Poder de inchamento e solubilidade de amido de arroz submetido ao tratamento térmico com baixa umidade", Brazilian Journal of Food Technology, pp. 31-35, 2009.

[29] RÓZ, A. L., Preparação e caracterização de amidos termoplásticos, Tese (Doutorado em Ciências e Engenharia de Materiais), IFSC/USP, São Carlos, SP, Brasil, 2004.

[30] CAENN, R., DARLEY, H. C. H., GRAY, G. R., Composition and properties of drilling and completion fluids, $6^{\mathrm{a}}$ ed., Houston, Gulf Publishing Company, 2011.

[31] ALASKARI, M. K. G., TEYMOORI, R. N., "Effects of salinity, pH and temperature on CMC polymer and XC polymer performance", International Journal of Engineering Transactions B: Applications, v. 20, $\mathrm{n}$. 3, pp. 283-290, 2007. 
[32] AlbuQuerque, M. C. C., BARbosA, A. C. C. A., SOUSA, M. P., et al., "Aplicações de enzimas em atividades de exploração e produção de petróleo", Boletim Técnico da Petrobras, v. 54, n. 3, pp. 9-28, 2011.

[33] LEITE, R. S., Otimização de sistemas de fluidos aquosos altamente inibidos, Dissertação (Mestrado em Ciência e Engenharia de Materiais), PPGCEMat/UFCG, Campina Grande, PB, Brasil, 2014.

[34] BLKOOR, S. O., FATTAH, K. A., "The influence of XC-polymer on drilling fluid filter cake properties and formation damage", Journal of Petroleum \& Environmental Biotechnology, v. 4, n. 5, pp. 1-10, 2013.

\section{ORCID}

Sarah Suelen Simões Silva https://orcid.org/0000-0003-2962-7336

Karine Castro Nóbrega https://orcid.org/0000-0003-4298-4178

Luciana Viana Amorim https://orcid.org/0000-0002-8207-901X

Hélio de Lucena Lira https://orcid.org/0000-0002-1527-9935 